\title{
Factoring in embedding dimension three numerical semigroups
}

\author{
F. Aguiló-Gost* \\ Dept. Matemàtica Aplicada IV \\ U. Politèctina de Catalunya, Barcelona, Spain \\ matfag@ma4.upc.edu
}

\author{
P. A. García-Sánchez ${ }^{\dagger}$ \\ Depto. de Álgebra \\ U. de Granada, E-18071 Granada, Spain \\ pedro@ugr.es
}

Submitted: Jan 5, 2010; Accepted: Sep 27, 2010; Published: Oct 15, 2010

Mathematics Subject Classifications: 05C90, 11D07, 11D45, 11P21

\begin{abstract}
Let us consider a 3-numerical semigroup $S=\langle a, b, N\rangle$. Given $m \in S$, the triple $(x, y, z) \in \mathbb{N}^{3}$ is a factorization of $m$ in $S$ if $x a+y b+z N=m$. This work is focused on finding the full set of factorizations of any $m \in S$ and as an application we compute the catenary degree of $S$. To this end, we relate a 2D tessellation to $S$ and we use it as a main tool.
\end{abstract}

\section{Introduction}

Let us denote the set of non negative integers by $\mathbb{N}$. A 3-numerical semigroup is the set $S=\langle a, b, N\rangle=\{x a+y b+z N \mid x, y, z \in \mathbb{N}\}$ with $1<a<b<N$ and $\operatorname{gcd}(a, b, N)=1$, such that the set $\{a, b, N\}$ is the minimal set of generators of $S$. The set $\bar{S}=\mathbb{N} \backslash S$ has finite cardinality with Frobenius' number $\mathfrak{f}(S)=\max \bar{S}$. The Apéry set of $S$ with respect to $m$ is the set $\operatorname{Ap}(m ; S)=\{s \in S \mid s-m \notin S\}$. This set acts like a boundary between elements that can be factored in $S$ and those that can not, inside each equivalence class modulo $m$ (the reader can find in [19] an introduction to numerical semigroups).

A factorization of $m \in S$ is a triple $(x, y, z) \in \mathbb{N}^{3}$ such that $x a+y b+z N=m$. Let us denote $\mathcal{F}(m ; S)=\left\{(x, y, z) \in \mathbb{N}^{3} \mid x a+y b+z N=m\right\}$ and $\mathrm{d}(m ; S)=|\mathcal{F}(m ; S)|$, also known as the denumerant of $m$ in $S$. See [17] for an exhaustive view of related results.

Example 1 For $S=\langle 3,5,7\rangle$ we have $\mathfrak{f}(S)=4$ and $\operatorname{Ap}(7 ; S)=\{0,3,5,6,8,9,11\}$. Consider $m=15$, we have $\mathrm{d}(m ; S)=3$ and $\mathcal{F}(m ; S)=\{(5,0,0),(0,3,0),(1,1,1)\}$.

${ }^{*}$ Work supported by MCYT ref. MTM2008-06620-C03-01/MTM and the Catalan Research Council under the projects DURSI 2005SGR00256 and 2009SGR1387.

${ }^{\dagger}$ Work supported by MCYT ref. MTM2007-62346 and MTM2010-15595 
Let $(x, y, z) \in \mathcal{F}(m ; S)$. The length of $(x, y, z)$ is $|(x, y, z)|=x+y+z$. For $(x, y, z),\left(x^{\prime}, y^{\prime}, z^{\prime}\right) \in \mathbb{N}^{3}$, write

$$
\operatorname{gcd}\left((x, y, z),\left(x^{\prime}, y^{\prime}, z^{\prime}\right)\right)=\left(\min \left\{x, x^{\prime}\right\}, \min \left\{y, y^{\prime}\right\}, \min \left\{z, z^{\prime}\right\}\right) .
$$

Given $\varpi, \varpi^{\prime} \in \mathbb{N}^{3}$, define

$$
\operatorname{dist}\left(\varpi, \varpi^{\prime}\right)=\max \left\{\left|\varpi-\operatorname{gcd}\left(\varpi, \varpi^{\prime}\right)\right|,\left|\varpi^{\prime}-\operatorname{gcd}\left(\varpi, \varpi^{\prime}\right)\right|\right\},
$$

to be the distance between $\varpi$ and $\varpi^{\prime}$ (see [13, Proposition 1.2.5] for a list of basic properties concerning the distance). Every $\varpi \in \mathbb{Z}^{3}$ can be uniquely expressed as $\varpi=\varpi^{+}-\varpi^{-}$, with $\varpi^{+}, \varpi^{-} \in \mathbb{N}^{3}$ and $\varpi^{+} \cdot \varpi^{-}=0$ (the dot product). Define the norm of $\varpi$ as

$$
\|\varpi\|=\max \left\{\left|\varpi^{+}\right|,\left|\varpi^{-}\right|\right\} .
$$

Observe that for $\varpi, \varpi^{\prime} \in \mathbb{N}^{3}$,

$$
\operatorname{dist}\left(\varpi, \varpi^{\prime}\right)=\left\|\varpi-\varpi^{\prime}\right\| \text {. }
$$

Given $m \in S$ and $\varpi, \varpi^{\prime} \in \mathcal{F}(m ; S)$, an $N$-chain of factorizations from $\varpi$ to $\varpi^{\prime}$ is a sequence $\varpi_{0}, \ldots, \varpi_{k} \in \mathcal{F}(m ; S)$ such that $\varpi_{0}=\varpi, \varpi_{k}=\varpi^{\prime}$ and $\operatorname{dist}\left(\varpi_{i}, \varpi_{i+1}\right) \leqslant N$ for all $i$. The catenary degree of $m, \mathrm{c}(m)$, is the minimal $N \in \mathbb{N} \cup\{\infty\}$ such that for any two factorizations $\varpi, \varpi^{\prime} \in \mathcal{F}(m ; S)$, there is an $N$-chain from $z$ to $z^{\prime}$. The catenary degree of $S, \mathrm{c}(S)$, is defined by

$$
\mathrm{c}(S)=\sup \{\mathrm{c}(m) \mid m \in S\} .
$$

In this work we give an expression for $\mathrm{d}(m ; S), \mathcal{F}(m ; S)$ and $\mathrm{c}(S)$ for any given 3numerical semigroup $S$ and $m \in \mathbb{N}$. As a direct consequence, we obtain expressions for $\mathrm{d}(m ;\langle a, b\rangle)$ and $\mathcal{F}(m ;\langle a, b\rangle)(1<a<b$ and $\operatorname{gcd}(a, b)=1)$. Several applications of these results are given as well. Some results of this work (with no proofs) were presented at the EUROCOMB'09 and can be found in [2].

Numerical computations have been done using the package numericalsgps implemented in GAP $[12,9]$ and several local programs implemented in Mathematica V6.0 [20]. Each package will be cited when needed.

\section{Some tools from Graph Theory}

From now on, we denote the equivalence class of $m$ modulo $N$ by $[m]_{N}$ and the element $m_{N} \in[m]_{N}$ is such that $m_{N} \in\{0,1, \ldots, N-1\}$. Given $1 \leqslant a<b<N$ with $\operatorname{gcd}(a, b, N)=$ 1, a weighted double-loop digraph, $G=G(N ; a, b ; \mathfrak{a}, \mathfrak{b})=G(V, E)$, is a directed graph with set of vertices $V=\mathbb{Z}_{N}=\left\{[0]_{N}, \ldots,[N-1]_{N}\right\}$ and set of weighted directed arcs

$$
A=\left\{[m]_{N} \stackrel{\mathfrak{a}}{\rightarrow}[m+a]_{N},[m]_{N} \stackrel{\mathfrak{b}}{\rightarrow}[m+b]_{N} \mid[m]_{N} \in V\right\} .
$$

The values $a$ and $b$ are the steps of $G$. The weights are given by $\mathfrak{a}$, for arcs defined by step $a$, and $\mathfrak{b}$ for the step $b$. 

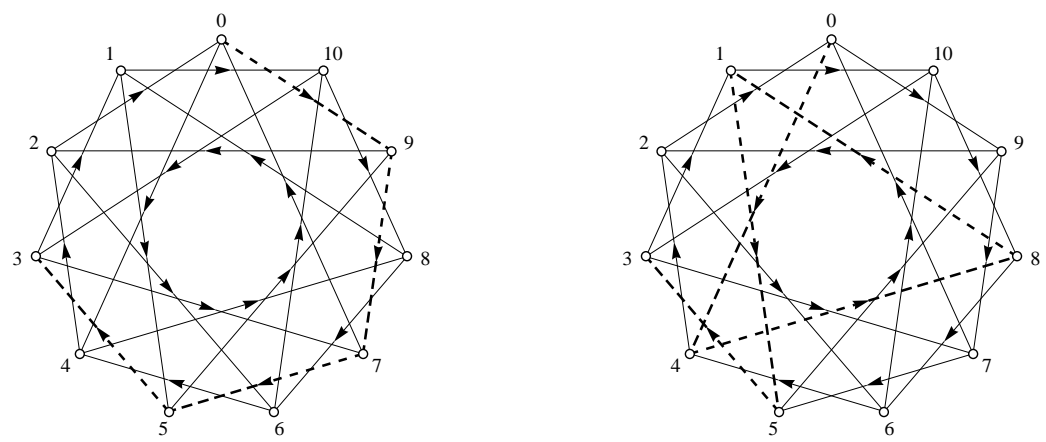

Figure $1: G(11 ; 4,9 ; \mathfrak{a}, \mathfrak{b})$ and short paths from $[0]_{11}$ to $[3]_{11}$, for $\mathfrak{a}=\mathfrak{b}=1$ and $\mathfrak{a}=4, \mathfrak{b}=9$

The length of a (directed) path between a pair of vertices is the sum of the weights of his arcs. The distance between vertices $v_{1}$ and $v_{2}$, $\operatorname{dist}_{G}\left(v_{1}, v_{2}\right)$, is the minimum length of all paths from $v_{1}$ to $v_{2}$ in $G$. Figure 1 shows weighted paths of minimum length joining $[0]_{11}$ and $[3]_{11}$ in $G(11 ; 4,9 ; \mathfrak{a}, \mathfrak{b})$ for $\mathfrak{a}=\mathfrak{b}=1$ (with length 4 ) and for $\mathfrak{a}=4, \mathfrak{b}=9$ (with length 25$)$, respectively. In this figure, the vertex $[m]_{11}$ has been denoted by $m$, for each $m=0, \ldots, 10$. Therefore, $\operatorname{dist}_{G}\left([0]_{11},[3]_{11}\right)=4$ for $\mathfrak{a}=\mathfrak{b}=1$ and $\operatorname{dist}_{G}\left([0]_{11},[3]_{11}\right)=25$ when $\mathfrak{a}=4, \mathfrak{b}=9$.

When using the particular weights $\mathfrak{a}=a$ and $\mathfrak{b}=b$, that is, when considering the graph $G(N ; a, b ; a, b)$, the distance $\operatorname{dist}_{G}\left([0]_{N},[m]_{N}\right)$ can be thought as the minimum element in $[m]_{N} \cap S$, where $S=\langle a, b, N\rangle$, not necessarily 3-generated. Thus we have

$$
\left\{\operatorname{dist}_{G}\left([0]_{N},[0]_{N}\right), \operatorname{dist}_{G}\left([0]_{N},[1]_{N}\right), \ldots, \operatorname{dist}_{G}\left([0]_{N},[N-1]_{N}\right)\right\}=\operatorname{Ap}(N ; S) .
$$

A well known geometrical approach of weighted double-loops digraphs will be used here for our purposes: L-shaped tiles related to these digraphs. It has been shown that each digraph can be linked to a plane periodical tessellation, generated by one tile of area $N$ that looks like an L-shape. In this approach, each vertex $[m]_{N}$ of $G$ is related to one unique unit square $(i, j)$, inside the L-shape, such that $i a+j b \equiv m(\bmod N)$. See $[21,18,11,8]$ for more details.

\begin{tabular}{|c|c|c|c|c|c|c|c|c|}
\hline 6 & 10 & 3 & 7 & 0 & 4 & 8 & 1 & 5 \\
\hline 8 & 1 & 5 & 9 & 2 & 6 & 10 & 3 & 7 \\
\hline 10 & 3 & 7 & 0 & 4 & 8 & 1 & 5 & 9 \\
\hline 1 & 5 & 9 & 2 & 6 & 10 & 3 & 7 & 0 \\
\hline 3 & 7 & 0 & 4 & 8 & 1 & 5 & 9 & 2 \\
\hline 5 & 9 & 2 & 6 & 10 & 3 & 7 & 0 & 4 \\
\hline 7 & 0 & 4 & 8 & 1 & 5 & 9 & 2 & 6 \\
\hline 9 & 2 & 6 & 10 & 3 & 7 & 0 & 4 & 8 \\
\hline 0 & 4 & 8 & 1 & 5 & 9 & 2 & 6 & 10 \\
\hline
\end{tabular}

\begin{tabular}{|c|c|c|c|c|c|c|c|c|}
\hline 6 & 10 & 3 & 7 & 0 & 4 & 8 & 1 & 5 \\
\hline 8 & 1 & 5 & 9 & 2 & 6 & 10 & 3 & 7 \\
\hline 10 & 3 & 7 & 0 & 4 & 8 & 1 & 5 & 9 \\
\hline 1 & 5 & 9 & 2 & 6 & 10 & 3 & 7 & 0 \\
\hline 3 & 7 & 0 & 4 & 8 & 1 & 5 & 9 & 2 \\
\hline 5 & 9 & 2 & 6 & 10 & 3 & 7 & 0 & 4 \\
\hline 7 & 0 & 4 & 8 & 1 & 5 & 9 & 2 & 6 \\
\hline 9 & 2 & 6 & 10 & 3 & 7 & 0 & 4 & 8 \\
\hline 0 & 4 & 8 & 1 & 5 & 9 & 2 & 6 & 10 \\
\hline
\end{tabular}

Figure 2: Minimum distance diagrams related to $G(11 ; 4,9 ; 1,1)$ and $G(11 ; 4,9 ; 4,9)$ 
When each vertex inside the L-shape is located at minimum distance from $[0]_{N}$, then this L-shape is called Minimum Distance Diagram. Tessellations generated by L-shapes which are also minimum distance diagrams of $G(11 ; 4,9 ; 1,1)$ and $G(11 ; 4,9 ; 4,9)$ are depicted in Figure 2, respectively; the minimum paths of Figure 1 are included in each related L-shape.
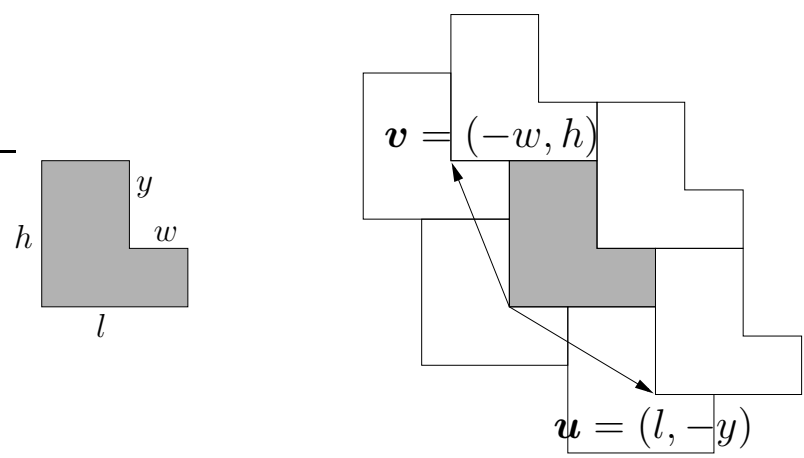

Figure 3: Generic lenghts of an L-shape and its related tessellation

L-shaped tiles will be denoted by their lenghts $L(l, h, w, y), \operatorname{gcd}(l, h, w, y)=1, l h-$ $w y=N, 0 \leqslant w<l$ and $0 \leqslant y<h$ as it is shown in Figure 3. Its related tessellation is generated by the pair of vectors $\boldsymbol{u}=(l,-y)$ and $\boldsymbol{v}=(-w, h)$. Thus, the distribution of zeros (or any other equivalence class modulo $N$ ) in the plane is defined by $\boldsymbol{u}$ and $\boldsymbol{v}$, or equivalently, the following conditions are fulfilled

$$
\left\{\begin{aligned}
l a-y b & \equiv 0(\bmod N) \\
-w a+h b & \equiv 0(\bmod N) .
\end{aligned}\right.
$$

There are double-loop digraphs that have linked minimum distance diagrams that are rectangles (that is, $w y=0$ ). Rectangle-shaped tiles are considered degenerated L-shapes, see [5]. The L-shaped minimum distance diagrams of Figure 2 are denoted by $L(4,5,3,3)$ and $L(5,3,4,1)$, respectively. The following known result characterizes the L-shapes that are also minimum distance diagrams of $G(N ; a, b ; a, b)$.

Theorem 1 ([3]) Let us consider the double-loop digraph $G=G(N ; a, b ; a, b)$. Let us assume that $\mathcal{H}=L(l, h, w, y), \operatorname{gcd}(l, h, w, y)=1$, is related to $G$. Then $\mathcal{H}$ is also a minimum distance diagram for $G$ if and only if $l a \geqslant y b$ and $h b \geqslant w a$.

Definition 1 Let be $S=\langle a, b, N\rangle$, with $1<a<b<N$ and $\operatorname{gcd}(a, b, N)=1$, a numerical semigroup. We say that the tile $\mathcal{H}=L(l, h, w, y)$ is related to $S$ if $\mathcal{H}$ is a minimum distance diagram of the weighted double-loop $G(N ; a, b ; a, b)$.

Let us consider $S=\langle a, b, N\rangle$ and the plane tessellation generated by one related Lshaped tile $\mathcal{H}$. Assume that each unit square $(i, j) \in \mathbb{N}^{2}$ is labelled by $i a+j b$. Let us consider the (unique) L-shape in this tessellation containing the value 0 . Then the squares inside $\mathcal{H}$ are labelled by $\left\{\operatorname{dist}_{G}\left([0]_{N},[0]_{N}\right), \ldots, \operatorname{dist}_{G}\left([0]_{N},[N-1]_{N}\right)\right\}$, that is, the set $\operatorname{Ap}(N ; S)$ is encoded by $\mathcal{H}$. 


\begin{tabular}{|c|c|c|c|c|c|c|c|c|}
\hline 45 & 49 & 53 & 57 & 61 & 65 & 69 & 73 & 77 \\
\hline 36 & 40 & 44 & 48 & 52 & 56 & 60 & 64 & 68 \\
\hline 27 & 31 & 35 & 39 & 43 & 47 & 51 & 55 & 59 \\
\hline 18 & 22 & 26 & 30 & 34 & 38 & 42 & 46 & 50 \\
\hline 9 & 13 & 17 & 21 & 25 & 29 & 33 & 37 & 41 \\
\hline 0 & 4 & 8 & 12 & 16 & 20 & 24 & 28 & 32 \\
\hline
\end{tabular}

Figure 4: $\operatorname{Ap}(11 ;\langle 4,9,11\rangle)$ encoded by $\mathcal{H}=L(5,3,4,1)$ in grey

Example 2 Figure 4 shows a piece of the first quadrant of the tessellated squared plane. This tessellation is given by the tile $\mathcal{H}=L(5,3,4,1)$ related to $S=\langle 4,9,11\rangle$. Each unit square $(i, j)$ is labelled with the value $4 i+9 j$. The $(0,0)$ unit square is located inside the gray L-shaped tile and it is labelled with 0 . In this case we have $\operatorname{Ap}(11 ; S)=$ $\{0,4,8,9,12,13,16,17,18,21,25\}$.

Lemma 1 Given any $S=\langle a, b, N\rangle$ and $m \in S$, let be $\left(s, t, z_{0}\right) \in \mathcal{F}(m ; S)$ with $z_{0}=$ $\max \{z \mid(x, y, z) \in \mathcal{F}(m ; S)\}$. Let $\mathcal{H}$ be a tile related to $S$ that encodes $A p(N ; S)$, then there is a unit square with coordinates $\left(x_{0}, y_{0}\right)$, inside $\mathcal{H}$, such that $\left(x_{0}, y_{0}, z_{0}\right) \in \mathcal{F}(m ; S)$.

Proof: Let us consider $(x, y, z) \in \mathcal{F}(m ; S)$, that is, $x a+y b+z N=m$. Then $x a+y b \equiv$ $m(\bmod N)$, that is, $x a+y b \in[m]_{N} \cap \mathbb{N}$. From the fact that $\mathcal{H}$ is a minimum distance diagram of $G(N ; a, b ; a, b)$, there is a unit square with coordinates $\left(x_{0}, y_{0}\right) \in \mathcal{H}$ such that $x_{0} a+y_{0} b=\min \left([m]_{N} \cap \mathbb{N}\right)=m^{\prime}$. Thus, in the factorization

$$
x_{0} a+y_{0} b+\frac{m-m^{\prime}}{N} N=m
$$

we have $z_{0}=\frac{m-m^{\prime}}{N} N=\max \{z \mid(x, y, z) \in \mathcal{F}(m ; S)\}$.

Definition 2 (basic coordinates) Let be $m \in \mathbb{N}$. Let us assume that $\mathcal{H}$ is related to $S$ and $\left(x_{0}, y_{0}\right)$ are the coordinates of the (unique) unit square inside $\mathcal{H}$ with label $[m]_{N}$. We call $\left(x_{0}, y_{0}\right)$ the basic coordinates of $m$ with respect to $\mathcal{H}$.

Proposition 1 Let be $\mathcal{H}$ an L-shaped tile related to $S=\langle a, b, N\rangle$. Let be $m \in \mathbb{N}$ and $\left(x_{0}, y_{0}\right)$ the basic coordinates of $m$ with respect to $\mathcal{H}$, then

$$
m \in S \Leftrightarrow x_{0} a+y_{0} b \leqslant m .
$$

Proof: If $m \in S$, then there is $(x, y, z) \in \mathbb{N}^{3}$ with $x a+y b+z N=m$. By Lemma 1 we have $x_{0} a+y_{0} b \leqslant x a+y b \leqslant m$.

If $x_{0} a+y_{0} b \leqslant m$, from the fact $x_{0} a+y_{0} b=m^{\prime} \in[m]_{N}$ we have $m-m^{\prime} \equiv 0(\bmod N)$. Thus, $z_{0}=\left(m-m^{\prime}\right) / N \in \mathbb{N}$. Since $x_{0} a+y_{0} b+z_{0} N=m$, we have $m \in S$.

From the computational point of view, Proposition 1 is a fast test when the coordinates $\left(x_{0}, y_{0}\right)$ can be efficiently computed. 
Definition 3 (basic factorization) Let be $m \in S$. Let us assume that $\mathcal{H}$ is related to $S$. Let $\left(x_{0}, y_{0}\right)$ be the basic coordinates of $m$ with respect to $\mathcal{H}$. The factorization $\left(x_{0}, y_{0}, z_{0}\right)$ given by Lemma 1 is called the basic factorization of $m$ with respect to $\mathcal{H}$.

Example 3 Let us consider $S=\left\langle 3^{5}, 5^{5}, 7^{5}\right\rangle$ and $m=\mathfrak{f}(S)+1=190464$. A related tile is given by the L-shape of lenghts $\mathcal{H}=L(521,37,130,19)$ and the basic coordinates are $\left(x_{0}, y_{0}\right)=(7,12)$. Thus, the basic factorization is $(7,12,9)$.

Note that for any $m \in \mathbb{N}$, it is well known, and easy to prove, that there exist unique $\alpha \in \operatorname{Ap}(N ; S)$ and $k \in \mathbb{Z}$ such that $m=\alpha+k N$, and $m \in S$ if and only if $\alpha \leqslant m$ (equivalently $k \geqslant 0$ ). Proposition 1 is a translation of this fact to the tessellation of the plane (by $\mathcal{H}$ ) related to $S$.

\section{Factorizations}

Given $S=\langle a, b, N\rangle, m \in S$ and $(x, y, z) \in \mathcal{F}(m ; A)$, the value $z$ is determined by $x$ and $y$ in the identity $x a+y b+z N=m$. Therefore, the problem of finding the set $\mathcal{F}(m ; S)$ is a two dimensional one.

Let us consider a tile $\mathcal{H}$ related to $S$. We search the first quadrant with the help of the tessellation by $\mathcal{H}$ to find all the pairs $(x, y) \in \mathbb{N}^{2}$ with $(x, y, z) \in \mathcal{F}(m ; S)$ for some $z \in \mathbb{N}$. In fact, due to Lemma 1 , we have

$$
(x, y, z) \in \mathcal{F}(m ; S) \Leftrightarrow(x, y) \in\left(x_{0}, y_{0}\right)+\langle\boldsymbol{u}, \boldsymbol{v}\rangle_{\mathbb{N}} \text { and } x a+y b \leqslant m
$$

where $\left(x_{0}, y_{0}\right)$ are the basic coordinates of $m$ and $\langle\boldsymbol{u}, \boldsymbol{v}\rangle_{\mathbb{N}}=\{\alpha \boldsymbol{u}+\beta \boldsymbol{v} \mid \alpha, \beta \in \mathbb{N}\}$.

Henceforth we will denote $\boldsymbol{e}=\boldsymbol{u}+\boldsymbol{v}=(l-w, h-y) \in \mathbb{N}^{2}$, where $\boldsymbol{u}$ and $\boldsymbol{v}$ are the generating vectors of the tessellation related to $S$.

Definition 4 (Gain fuction) Let us define the gain function $\mathcal{G}: \mathbb{Z}^{2} \rightarrow \mathbb{Z}$, with $\mathcal{G}(x, y)=$ $x a+y b$.

By (1), we note that the gains of $\boldsymbol{u}, \boldsymbol{v}$ and $\boldsymbol{e}$ are multiples of $N$ :

$$
\begin{aligned}
& \mathcal{G}(\boldsymbol{u})=l a-y b=\delta N, \\
& \mathcal{G}(\boldsymbol{v})=-w a+h b=\theta N, \\
& \mathcal{G}(\boldsymbol{e})=(l-w) a+(h-y) b=(\delta+\theta) N .
\end{aligned}
$$

Lemma 2 For $\delta$ and $\theta$ defined above, we have $\delta \geqslant 0, \theta \geqslant 0$ and $\delta+\theta \neq 0$.

Proof: Theorem 1 assures $\delta \geqslant 0$ and $\theta \geqslant 0$. Thus, $\delta+\theta=0$ implies that both $\delta$ and $\theta$ are zero. Hence

$l a=y b, w a=h b \Rightarrow(l-w) a=(y-h) b \Rightarrow(l-w) a+(h-y) b=0 \Rightarrow l-w=h-y=0$.

Therefore $N=l h-w y=0$, a contradiction. 
In terms of the gain function, Proposition 1 implies $m \in S$ if and only if $\mathcal{G}\left(\left(x_{0}, y_{0}\right)\right) \leqslant$ $m$, where $\left(x_{0}, y_{0}\right)$ are the basic coordinates of $m$. Following this idea, we will do the search of factorizations from the starting point $\left(x_{0}, y_{0}\right)$ using $(2)$ and checking that the gain does not surpass the value of $m$.

From now on, we will denote $m^{\prime}=x_{0} a+y_{0} b$ and $z_{0}=\left(m-m^{\prime}\right) / N$.

Proposition 2 Given $k \in \mathbb{N}$, we have

$$
\mathcal{G}\left(\left(x_{0}, y_{0}\right)+k \boldsymbol{e}\right) \leqslant m \Leftrightarrow k \leqslant\left\lfloor\frac{z_{0}}{\delta+\theta}\right\rfloor .
$$

Proof: From $l-w \geqslant 0$ and $h-y \geqslant 0$, we have that $\left(x_{0}, y_{0}\right)+k \boldsymbol{e}$ belongs to the first quadrant for all natural value of $k$. Moreover

$$
\mathcal{G}\left(\left(x_{0}, y_{0}\right)+k \boldsymbol{e}\right)=\left[x_{0}+k(l-w)\right] a+\left[y_{0}+k(h-y)\right] b=m^{\prime}+k(\delta+\theta) N .
$$

Then

$$
\mathcal{G}\left(\left(x_{0}, y_{0}\right)+k \boldsymbol{e}\right) \leqslant m \Leftrightarrow m^{\prime}+k(\delta+\theta) N \leqslant m^{\prime}+z_{0} N \Leftrightarrow k \leqslant \frac{z_{0}}{\delta+\theta} .
$$

Definition 5 Let us denote $p_{k}=\left(x_{0}, y_{0}\right)+k \boldsymbol{e}=\left(x_{0}+k(l-w), y_{0}+k(h-y)\right)=\left(x_{k}, y_{k}\right)$, for $k=0, \ldots,\left\lfloor\frac{z_{0}}{\delta+\theta}\right\rfloor$.

Corollary 1 Let us consider $z_{k}=z_{0}-k(\delta+\theta)$ for each $k=0, \ldots,\left\lfloor\frac{z_{0}}{\delta+\theta}\right\rfloor$. Then $\left(x_{k}, y_{k}, z_{k}\right) \in$ $\mathcal{F}(m ; S)$.

Proof: By definition

$x_{k} a+y_{k} b+z_{k} N=x_{0} a+y_{0} b+z_{0} N+k(l-w) a+k(h-y) b-k(\delta+\theta) N=m+0=m$.

Proposition 3 For each $k=0, \ldots,\left\lfloor\frac{z_{0}}{\delta+\theta}\right\rfloor$, set

$$
S_{k}= \begin{cases}\left\lfloor\frac{y_{0}+k(h-y)}{y}\right\rfloor & \text { if } \delta=0, \\ \left\lfloor\frac{z_{0}-k(\delta+\theta)}{\delta}\right\rfloor & \text { if } y=0, \\ \min \left\{\left\lfloor\frac{y_{0}+k(h-y)}{y}\right\rfloor,\left\lfloor\frac{z_{0}-k(\delta+\theta)}{\delta}\right\rfloor\right\} & \text { if } \delta y \neq 0 .\end{cases}
$$

Then $p_{k}+s \boldsymbol{u} \in \mathbb{N}^{2}$ and $\mathcal{G}\left(p_{k}+s \boldsymbol{u}\right) \leqslant m$ only for $s=0, \ldots, S_{k}$.

Proof: $S_{k}$ is well defined because the case $\delta=y=0$ is not possible: if $\delta=0$, then $l a=y b ;$ if $y=0$ also, then $l=0$ and $N=l h-w y=0$, which is a contradiction.

We have $p_{k}+s \boldsymbol{u}=\left(x_{k}, y_{k}\right)+s(l,-y)=\left(x_{k}+s l, y_{k}-s y\right)=\left(x_{k, s}, y_{k, s}\right)$ and

$$
\mathcal{G}\left(p_{k}+s \boldsymbol{u}\right)=\mathcal{G}\left(p_{k}\right)+s \mathcal{G}(\boldsymbol{u})=m^{\prime}+k(\delta+\theta) N+s \delta N .
$$


If $y=0(\Rightarrow \delta \neq 0)$, we have $p_{k}+s \boldsymbol{u} \in \mathbb{N}^{2}$ for all $s \in \mathbb{N}$. Then

$$
\mathcal{G}\left(p_{k}+s \boldsymbol{u}\right) \leqslant m \Leftrightarrow m^{\prime}+k(\delta+\theta) N+s \delta N \leqslant m^{\prime}+z_{0} N \Leftrightarrow s \leqslant \frac{z_{0}-k(\delta+\theta)}{\delta} .
$$

If $\delta=0(\Rightarrow y \neq 0)$, then $\mathcal{G}\left(p_{k}+s \boldsymbol{u}\right) \leqslant m$ for all $s \in \mathbb{N}$ (by Proposition 2). Thus, we only have to check that $p_{k}+s \boldsymbol{u} \in \mathbb{N}^{2}$, that is, $y_{k}-s y \geqslant 0 \Rightarrow s \leqslant \frac{y_{k}}{y}=\frac{y_{0}+k(h-y)}{y}$.

If $\delta y \neq 0$, we have to check both conditions. Therefore $s \leqslant \min \left\{\frac{z_{0}-k(\delta+\theta)}{\delta}, \frac{y_{0}+k(h-y)}{y}\right\}$.

Definition 6 Let us denote $\left(x_{k, s}, y_{k, s}\right)=p_{k}+s \boldsymbol{u}=\left(x_{k}+s l, y_{k}-s y\right)$, for $k=0, \ldots,\left\lfloor\frac{z_{0}}{\delta+\theta}\right\rfloor$ and $s=0, \ldots, S_{k}$.

Corollary 2 Let be $z_{k, s}=z_{0}-k(\delta+\theta)-s \delta$ for $k=0, \ldots,\left\lfloor\frac{z_{0}}{\delta+\theta}\right\rfloor$ and $s=0, \ldots, S_{k}$. Then $\left(x_{k, s}, y_{k, s}, z_{k, s}\right) \in \mathcal{F}(m ; S)$.

Proof: By using Proposition 3, the proof follows as in Corollary 1.

Proposition 4 For each $k=0, \ldots,\left\lfloor\frac{z_{0}}{\delta+\theta}\right\rfloor$, set

$$
T_{k}= \begin{cases}\left\lfloor\frac{x_{0}+k(l-w)}{w}\right\rfloor & \text { if } \theta=0, \\ \left\lfloor\frac{z_{0}-k(\delta+\theta)}{\theta}\right\rfloor & \text { if } w=0, \\ \min \left\{\left\lfloor\frac{x_{0}+k(l-w)}{w}\right\rfloor,\left\lfloor\frac{z_{0}-k(\delta+\theta)}{\theta}\right\rfloor\right\} & \text { if } \theta w \neq 0 .\end{cases}
$$

Then $p_{k}+t \boldsymbol{v} \in \mathbb{N}^{2}$ and $\mathcal{G}\left(p_{k}+t \boldsymbol{v}\right) \leqslant m$ only for $t=1, \ldots, T_{k}$.

Proof: $T_{k}$ is well defined because the case $w=\theta=0$ is not possible: if $\theta=0$, then $w a=h b$; if $w=0$, then $h=0$ which implies $N=l h-w y=0$, a contradiction.

If $w=0(\Rightarrow \theta \neq 0)$, then $p_{k}+t \boldsymbol{v} \in \mathbb{N}^{2}$ for all $t \in \mathbb{N}$. The condition $\mathcal{G}\left(p_{k}+t \boldsymbol{v}\right) \leqslant m$ is equivalent to

$$
m^{\prime}+k(\delta+\theta) N+t \theta N \leqslant m^{\prime}+z_{0} N \Leftrightarrow t \leqslant \frac{z_{0}-k(\delta+\theta)}{\theta} .
$$

If $\theta=0(\Rightarrow w \neq 0)$, then $\mathcal{G}\left(p_{k}+t \boldsymbol{v}\right)=\mathcal{G}\left(p_{k}\right) \leqslant m$ for all $t \in \mathbb{N}$. Now we have to check that $p_{k}+t \boldsymbol{v} \in \mathbb{N}^{2}$; this condition is true if and only if $x_{k}-t w \geqslant 0 \Rightarrow t \leqslant \frac{x_{k}}{w}=\frac{x_{0}+k(l-w)}{w}$. If $\theta w \neq 0$, both conditions have to be fulfilled, that is, $t \leqslant \min \left\{\frac{z_{0}-k(\delta+\theta)}{\theta}, \frac{x_{0}+k(l-w)}{w}\right\}$.

Definition 7 Let us denote $\left(\bar{x}_{k, t}, \bar{y}_{k, t}\right)=p_{k}+t \boldsymbol{v}=\left(x_{k}-t w, y_{k}+t h\right)$, for $k=0, \ldots,\left\lfloor\frac{z_{0}}{\delta+\theta}\right\rfloor$ and $t=1, \ldots, T_{k}$.

Corollary 3 Set $\bar{z}_{k, t}=z_{0}-k(\delta+\theta)-t \theta$ for $k=0, \ldots,\left\lfloor\frac{z_{0}}{\delta+\theta}\right\rfloor$ and $t=1, \ldots, T_{k}$. Then $\left(\bar{x}_{k, t}, \bar{y}_{k, t}, \bar{z}_{k, t}\right) \in \mathcal{F}(m ; S)$.

Proof: By using Proposition 4, the proof follows as in Corollary 1. 
Lemma 3 The triple $(x, y, z) \in \mathcal{F}(m ; S)$ if and only if $(x, y) \in\left(x_{0}, y_{0}\right)+\langle\boldsymbol{e}, \boldsymbol{u}\rangle_{\mathbb{N}} \cup\left(x_{0}, y_{0}\right)+$ $\langle\boldsymbol{e}, \boldsymbol{v}\rangle_{\mathbb{N}}$ and $\mathcal{G}(x, y) \leqslant m$.

Proof: It is a direct consequence of $\langle\boldsymbol{u}, \boldsymbol{v}\rangle_{\mathbb{N}}=\langle\boldsymbol{e}, \boldsymbol{u}\rangle_{\mathbb{N}} \cup\langle\boldsymbol{e}, \boldsymbol{v}\rangle_{\mathbb{N}}$ and (2).

Corollary 4 Each element $(x, y, z) \in \mathcal{F}(m ; S)$ has the expression $(x, y)=p_{k}+$ su or $(x, y)=p_{k}+t \boldsymbol{v}$ for some $0 \leqslant k \leqslant\left\lfloor\frac{z_{0}}{\delta+\theta}\right\rfloor$ and $0 \leqslant s \leqslant S_{k}$ or $1 \leqslant t \leqslant T_{k}$, and does not admit both expressions at the same time.

Proof: It is a direct consequence of Propositions 2, 3 and 4 and Lemma 3: $\{\boldsymbol{u}, \boldsymbol{v}\},\{\boldsymbol{e}, \boldsymbol{u}\}$ and $\{\boldsymbol{e}, \boldsymbol{v}\}$ are sets of linearly independent vectors, then

- $k \boldsymbol{e}+s \boldsymbol{u}=k^{\prime} \boldsymbol{e}+s^{\prime} \boldsymbol{u}$ implies $k=k^{\prime}$ and $s=s^{\prime}$.

- $k \boldsymbol{e}+t \boldsymbol{v}=k^{\prime} \boldsymbol{e}+t^{\prime} \boldsymbol{v}$ implies $k=k^{\prime}$ and $t=t^{\prime}$.

- Finally, we have $k \boldsymbol{e}+s \boldsymbol{u} \neq l \boldsymbol{e}+\boldsymbol{t} \boldsymbol{v}$ for all $0 \leqslant k, l \leqslant\left\lfloor\frac{z_{0}}{\delta+\theta}\right\rfloor, 0 \leqslant s \leqslant S_{k}$ and $1 \leqslant t \leqslant T_{l}$ : actually, if $k \boldsymbol{e}+s \boldsymbol{u}=l \boldsymbol{e}+t \boldsymbol{v}$, then $(k+s) \boldsymbol{u}+k \boldsymbol{v}=l \boldsymbol{u}+(l+t) \boldsymbol{v}$; hence $k+s=l$ and $k=l+t$. Therefore, we have $t+s=0$, a contradiction $(s \geqslant 0, t \geqslant 1)$.

Theorem 2 Let be $m \in S$ and $\left(x_{0}, y_{0}, z_{0}\right)$ a basic factorization of $m$, then

$$
\begin{aligned}
& \mathcal{F}(m ; S)=\bigcup_{k=0}^{\left\lfloor\frac{z_{0}}{\delta+\theta}\right\rfloor}\left(\bigcup_{s=0}^{S_{k}}\left\{\left(x_{k, s}, y_{k, s}, z_{k, s}\right)\right\} \cup \bigcup_{t=1}^{T_{k}}\left\{\left(\bar{x}_{k, t}, \bar{y}_{k, t}, \bar{z}_{k, t}\right)\right\}\right), \\
& \mathrm{d}(m ; S)=1+\left\lfloor\frac{z_{0}}{\delta+\theta}\right\rfloor+\sum_{k=0}^{\left\lfloor\frac{z_{0}}{\delta+\theta}\right\rfloor}\left(S_{k}+T_{k}\right) .
\end{aligned}
$$

Proof: From Propositions 2, 3 and 4 and Corollary 4, we have

$$
(x, y, z) \in \mathcal{F}(m ; S) \Leftrightarrow(x, y) \in \bigcup_{k=0}^{\left\lfloor\frac{z_{0}}{\delta+\theta}\right\rfloor}\left(\bigcup_{s=0}^{S_{k}}\left\{p_{k}+s \boldsymbol{u}\right\} \cup \bigcup_{t=1}^{T_{k}}\left\{p_{k}+t \boldsymbol{v}\right\}\right)
$$

When expanding this expression, $z$ is given by Corollary 2 or Corollary 3 for each $(x, y, z) \in$ $\mathcal{F}(m ; S)$ and all factorizations of both corollaries are different in view of Corollary 4.

For each $k$, there are $1+S_{k}$ factorizations of the form $\left(x_{k, s}, y_{k, s}\right)=p_{k}+s \boldsymbol{u}$ and $T_{k}$ factorizations of the form $\left(\bar{x}_{k, t}, \bar{y}_{k, t}\right)=p_{k}+t \boldsymbol{v}$. Therefore $\mathrm{d}(m ; S)=\sum_{k=0}^{\left\lfloor\frac{z_{0}}{\delta+\theta}\right\rfloor}\left(1+S_{k}+T_{k}\right)$, that yields the stated expression for the denumerant.

The search of factorizations can be thought of in $\mathbb{N}^{2}$, through the tessellation by $\mathcal{H}$, as a rooted directed tree with root $\left(x_{0}, y_{0}\right)$; the arcs are given by $\boldsymbol{e}, \boldsymbol{u}$ and $\boldsymbol{v}$ according to the rules of the full parameterization of Theorem 2. This geometrical approach is visualized in the following example. 


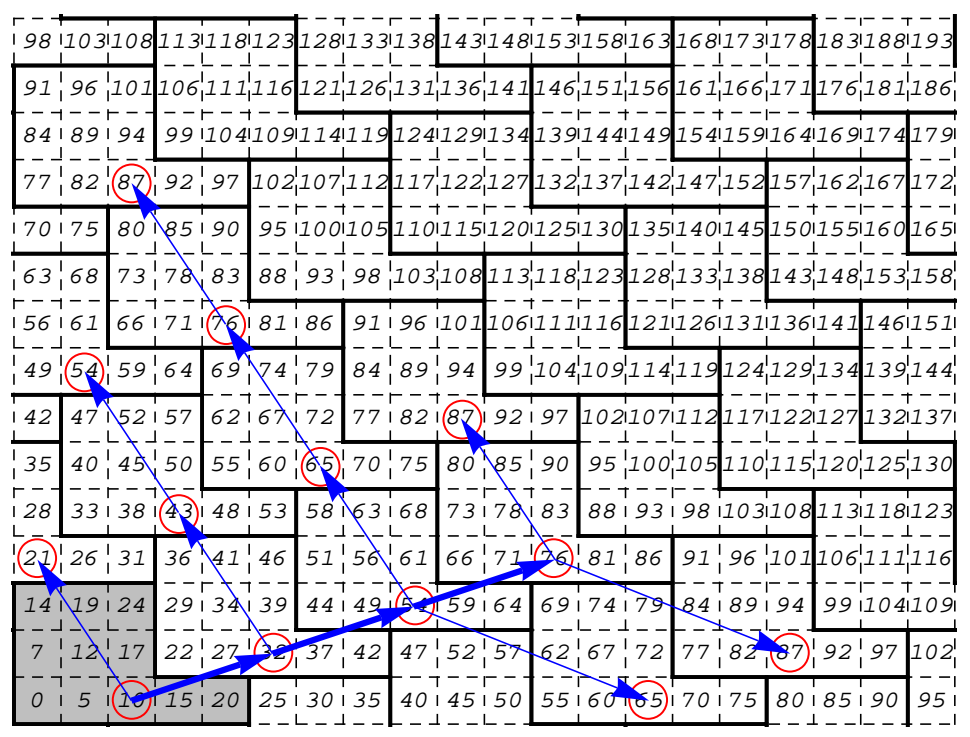

Figure 5: Tree-like approach of $\mathcal{F}(87 ;\{5,7,11\})$

Example 4 Let us consider $S=\langle 5,7,11\rangle$ and $m=87$. Then, a L-shaped tile is $\mathcal{H}=$ $L(5,3,2,2)$ and $\boldsymbol{u}=(5,-2), \boldsymbol{v}=(-2,3), \boldsymbol{e}=(3,1)$. Therefore, we have $\delta=\theta=1$, $0 \leqslant k \leqslant 3,\left\{S_{k}\right\}_{k=0}^{3}=\{1,2,3,1\}$ and $\left\{T_{k}\right\}_{k=0}^{3}=\{0,0,1,1\}$. Thus, we have

$$
\begin{aligned}
\mathrm{d}(87 ; S)= & 13, \\
\mathcal{F}(87 ; S)= & \{(2,0,7),(0,3,6),(5,1,5),(3,4,4),(1,7,3),(8,2,3),(13,0,2), \\
& (6,5,2),(4,8,1),(2,11,0),(11,3,1),(16,1,0),(9,6,0)\} .
\end{aligned}
$$

The tree-like visualization of $\mathcal{F}(87 ; S)$ is depicted in Figure 5.

As a direct consequence of Theorem 2, factorizations of 2-numerical semigroups can also be found.

Lemma $4(x, y) \in \mathcal{F}(m ;\langle a, b\rangle) \Leftrightarrow(x, y, 0) \in \mathcal{F}(m ;\langle a, b, a+b\rangle)$.

Proof: It is a direct fact with no need of proof. However it can be emphasized the fact that any $(x, y, z) \in \mathcal{F}(\langle a, b, a+b\rangle)$ with $z \neq 0$ is equivalent to $(x+z, y+z, 0)$ when considered in $\mathcal{F}(m ;\langle a, b\rangle)$.

Corollary 5 Let us consider $S=\langle a, b\rangle$, with $1<a<b$ and $\operatorname{gcd}(a, b)=1$. Take $m \in S$. Let $\left(x_{0}, y_{0}, z_{0}\right)$ be the basic factorization of $m$ with respect to $\mathcal{H}=L(b+1, a, b, a-1)$ related to $\langle a, b, a+b\rangle$. Then

$$
d(m ;\langle a, b\rangle)=z_{0}-\left\lceil\frac{z_{0}(a-1)-y_{0}}{a}\right\rceil+1+\left\lfloor\frac{x_{0}+z_{0}}{b}\right\rfloor
$$


and $\mathcal{F}(m ;\langle a, b\rangle)$ is given by

$$
\bigcup_{k=\left\lceil\frac{z_{0}(a-1)-y_{0}}{a}\right\rceil}^{z_{0}}\left\{\left(x_{0}+z_{0}(b+1)-k b, y_{0}-z_{0}(a-1)+k a\right)\right\} \cup \bigcup_{t=1}^{\left\lfloor\frac{x_{0}+z_{0}}{b}\right\rfloor}\left\{\left(x_{0}+z_{0}-t b, y_{0}+z_{0}+t a\right)\right\} .
$$

Proof: Let us consider $\langle a, b, a+b\rangle$. By Lemma 4,

$$
\mathcal{F}(m ;\langle a, b\rangle)=\{(x, y) \mid(x, y, 0) \in \mathcal{F}(m ;\langle a, b, a+b\rangle)\} .
$$

Let us consider the tile is $\mathcal{H}=L(b+1, a, b, a-1)$ which, by Theorem 1 , is related to $\langle a, b, a+b\rangle$. Thus, we have $\boldsymbol{u}=(b+1,1-a), \boldsymbol{v}=(-b, a), \boldsymbol{e}=(1,1), \mathcal{G}(\boldsymbol{u})=\delta=1$ and $\mathcal{G}(\boldsymbol{v})=\theta=0$. Let $\left(x_{0}, y_{0}, z_{0}\right)$ be the basic factorization of $m$ with respect to $\mathcal{H}$. By Theorem $2, \mathcal{F}(m ;\langle a, b, a+b\rangle)=C_{1} \cup C_{2}$ with

$$
\begin{aligned}
C_{1} & =\bigcup_{k=0}^{z_{0}} \bigcup_{s=0}^{S_{k}}\left\{\left(x_{0}+k+s(b+1), y_{0}+k-s(a-1), z_{0}-k-s\right)\right\}, \\
C_{2} & =\bigcup_{k=0}^{z_{0}} \bigcup_{t=1}^{T_{k}}\left\{\left(x_{0}+k-t b, y_{0}+k+t a, z_{0}-k\right)\right\}, \\
S_{k} & =\min \left\{\left\lfloor\frac{y_{0}+k}{a-1}\right\rfloor, z_{0}-k\right\}, \\
T_{k} & =\left\lfloor\frac{x_{0}+k}{b}\right\rfloor .
\end{aligned}
$$

From their definitions, it is always true that $C_{1} \cap C_{2}=\emptyset$. Let us search triples $(x, y, 0)$-like in $C_{1}$ and $C_{2}$. In $C_{1}$, we have $z_{0}-k-s=0$ whenever $s=z_{0}-k$. From

$$
S_{k}=z_{0}-k \Leftrightarrow z_{0}-k \leqslant\left\lfloor\frac{y_{0}+k}{a-1}\right\rfloor \Leftrightarrow z_{0}-k \leqslant \frac{y_{0}+k}{a-1} \Leftrightarrow k \geqslant\left\lceil\frac{z_{0}(a-1)-y_{0}}{a}\right\rceil,
$$

and $\frac{z_{0}(a-1)-y_{0}}{a} \leqslant z_{0}$, we always have $\left\{(x, y) \mid(x, y, 0) \in C_{1}\right\} \neq \emptyset$ and

$$
\left\{(x, y) \mid(x, y, 0) \in C_{1}\right\}=\bigcup_{k=\left\lceil\frac{z_{0}(a-1)-y_{0}}{a}\right\rceil}^{z_{0}}\left\{\left(x_{0}+z_{0}(b+1)-k b, y_{0}-z_{0}(a-1)+k a\right)\right\} .
$$

In $C_{2}$, one have to take $k=z_{0}$. Hence,

$$
\left\{(x, y) \mid(x, y, 0) \in C_{2}\right\}=\bigcup_{t=1}^{\left\lfloor\frac{x_{0}+z_{0}}{b}\right\rfloor}\left\{\left(x_{0}+z_{0}-t b, y_{0}+z_{0}+t a\right)\right\}
$$

where this set is empty whenever $\left\lfloor\frac{x_{0}+z_{0}}{b}\right\rfloor=0$.

Clearly we have $\left|\left\{(x, y) \mid(x, y, 0) \in C_{1}\right\}\right|=z_{0}-\left\lceil\frac{z_{0}(a-1)-y_{0}}{a}\right\rceil+1$ and $\mid\{(x, y) \mid(x, y, 0) \in$ $\left.C_{2}\right\} \mid=\left\lfloor\frac{x_{0}+z_{0}}{b}\right\rfloor$. Now from $C_{1} \cap C_{2}=\emptyset$, the value of $\mathrm{d}(m ;\langle a, b\rangle)$ is the one stated above.

Expression (3) of Corollary 5 complements the well known expression of $\mathrm{d}(m ;\langle a, b\rangle)$ given by Popoviciu in [16], cited in [17, page 80]. 


\section{Catenary Degree}

In this section, all numerical semigroups $S=\langle a, b, N\rangle$ will be assumed to be 3-generated, that is the set $\{a, b, N\}$ is a minimal set of generators of $S$. Let $\mathcal{H}=L(l, h, w, y)$ be a tile related to $S$. Set

- $\alpha=(l,-y,-\delta)$,

- $\beta=(-w, h,-\theta)$,

- $\gamma=\alpha+\beta=(l-w, h-y,-(\delta+\theta))$.

Lemma 5 If $\mathcal{H}=L(l, h, w, y)$ is a tile related to $S$, then

$$
\|\alpha\|=l,\|\beta\|=\max \{h, w+\theta\},\|\gamma\|=l-w+h-y .
$$

Proof: Note that $l a=\delta N+y b$. Hence $l=\delta \frac{N}{a}+y \frac{b}{a} \geqslant \delta+y$. Thus $\max \{l, y+\delta\}=l$. Analogously, $(l-w) a+(h-y) b=(\delta+\theta) N$. This leads to $\delta+\theta=(l-w) \frac{a}{N}+(h-y) \frac{b}{N} \leqslant$ $(l-w)+(h-y)$. Consequently $\|\gamma\|=l-w+h-y$.

From the tree like shape described in Theorem 2 for the set $\mathcal{F}(m ; S)$, with $m \in S$, one easily deduces the following consequence.

Corollary 6 Let $\mathcal{H}=L(l, h, w, y)$ be a tile related to $S$, then

$$
\mathrm{c}(S) \leqslant \max \{l, w+\theta, h, l-w+h-y\} .
$$

Proof: Let $m \in S$ and take $\varpi \in \mathcal{F}(m ; S)$. Note that

- $\operatorname{dist}(\varpi, \varpi+\alpha)=\|\alpha\|=\max \{l, y+\delta\}$,

- $\operatorname{dist}(\varpi, \varpi+\beta)=\|\beta\|=\max \{h, w+\theta\}$,

- $\operatorname{dist}(\varpi, \varpi+\gamma)=\|\gamma\|=\max \{(l-w)+(h-y), \delta+\theta\}$.

From Theorem 2, we know that given two factorizations $\varpi, \varpi^{\prime} \in \mathcal{F}(m ; S)$, there exists a chain of factorizations $\varpi_{0}, \ldots, \varpi_{k}$ (it is just the path in the tree joining the nodes $\varpi$ and $\varpi^{\prime}$ ) such that $\varpi_{0}=\varpi, \varpi_{k}=\varpi^{\prime}$ and so that for every $i \in\{0, \ldots, k-1\}$, $\left\{\varpi_{i}, \varpi_{i+1}\right\}=\{\varpi, \varpi+\rho\}$, for some $\varpi \in \mathcal{F}(m ; S)$ and $\rho \in\{\alpha, \beta, \gamma\}$. The proof now follows easily from the above remarks and Lemma 5 .

Example 5 Let $S=\langle 10,13,21\rangle$. Then $(l, h, w, y, \delta, \theta)=(6,4,1,3,1,2)$. Hence the bound given in Corollary 6 is 6 , and the catenary degree of $S$ is also 6 (this computation can be performed by using [6], or the implementation of the algorithm presented there that is part of the numericalsgps GAP package; see [9]). 
We see next when the above bound is reached, and when it can be sharpened. Observe that if $\varpi \in \mathcal{F}(m ; S), \varpi+\gamma \in \mathcal{F}(m ; S)$, and either $\varpi+\alpha$ or $\varpi+\beta$ is in $\mathcal{F}(m ; S)$, then there are at least two paths connecting $\varpi$ and $\varpi+\gamma$. One is $\varpi, \varpi+\gamma$, and the other is either $\varpi, \varpi+\alpha, \varpi+\gamma$ or $\varpi, \varpi+\beta, \varpi+\gamma$. In the first case, clearly the distance between the two steps of the path is $\|\gamma\|$, whilst in the second case the distance between two consecutive links is bounded by $\max \{\|\alpha\|,\|\beta\|\}$. It may happen that $\|\gamma\|>\max \{\|\alpha\|,\|\beta\|\}$, and in this case the catenary degree of $m$ is at $\operatorname{most} \max \{\|\alpha\|,\|\beta\|\}$, whence the bound given above is not reached in this element. The same holds for $\|\alpha\|>\max \{\|\beta\|,\|\gamma\|\}$ and $\|\beta\|>\max \{\|\alpha\|,\|\gamma\|\}$.

The above idea can be formalized by using the concept of $\mathfrak{R}$-class. Given $m \in S$, two factorizations $\varpi$ and $\varpi^{\prime}$ of $m$ are $\mathfrak{R}$-related if there exists $\varpi_{1}, \ldots, \varpi_{n} \in \mathcal{F}(m ; S)$ such that $\varpi_{i} \cdot \varpi_{i+1} \neq 0$. According to [7], the catenary degree of $S$ is reached in elements with at least two $\mathfrak{R}$-classes. In a numerical semigroup generated by three integers, these elements are easy to determine. Define

$$
\begin{aligned}
& c_{a}=\min \{k \in \mathbb{N} \backslash\{0\} \mid k a \in\langle b, N\rangle\}, \\
& c_{b}=\min \{k \in \mathbb{N} \backslash\{0\} \mid k b \in\langle a, N\rangle\}, \\
& c_{N}=\min \{k \in \mathbb{N} \backslash\{0\} \mid k N \in\langle a, b\rangle\} .
\end{aligned}
$$

It can be easily deduced that $m \in S$ has more than one $\mathfrak{R}$-classes if and only if $m \in$ $\left\{c_{a} a, c_{b} b, c_{N} N\right\}$ (see for instance [14]). By [7, Theorem 3.1], in order to compute the catenary degree of an element with more than one $\mathfrak{R}$-class, it suffices to compute the minimum length of the factorizations in one $\mathfrak{R}$-class, and then take the maximum of these lengths among all $\mathfrak{R}$-classes. This is the idea used to prove the following property.

Proposition 5 Let $\mathcal{H}=L(l, h, w, y)$ be a tile related to $S$.

1) If $w=0$, then

$$
\mathrm{c}(S)=\max \{l, h\}
$$

2) If $w \neq 0$ and $y=0$, then

$$
c(S)= \begin{cases}\max \left\{w, l+\left\lfloor\frac{l}{w}\right\rfloor(h-w)\right\} & \text { if } \theta=0 \\ \max \{l, w+\theta, h\} & \text { otherwise. }\end{cases}
$$

3) If $w y \neq 0$, then

$$
c(S)= \begin{cases}\max \{l, l-w+h-y\} & \text { if } \delta=0, \\ \max \left\{w, l-y+\left\lfloor\frac{l}{w}\right\rfloor(h-w)\right\} & \text { if } \theta=0, \\ \max \{l, w+\theta, h, l-w+h-y\} & \text { otherwise. }\end{cases}
$$

Proof: The computations given below of $c_{a}, c_{b}$ and $c_{N}$ follow directly from Theorem 2 and the definition of $\alpha, \beta$ and $\gamma$. 
1) Observe that $w=0$ forces $\theta$ to be nonzero. In this setting $c_{a}=l$ and $c_{N}=\theta$.

If $\delta=0, c_{b}=y$. In view of [7, Theorem 3.1], in order to compute $\mathrm{c}(S)$, we must only compute the maximum of the catenary degrees of $c_{a} a\left(=c_{b} b\right)$ and $c_{N} N$. Clearly, $\mathcal{F}\left(c_{a} a ; S\right)=\{(l, 0,0),(0, y, 0)\}$. As $y>l, \mathrm{c}\left(c_{a} a\right)=l$. The $\mathfrak{R}$-classes of $\mathcal{F}\left(c_{N} N ; S\right)$ are $\{(0,0, \theta)\}$ and the one containing $(0, h, 0)$, which may also contain elements of the form $(i l, h-i y, 0)$, for some nonnegative integer $i$. The length of $(i l, h-i y, 0)$ is greater than or equal to $h$. Thus $(0, h, 0)$ is the element with least length in its $\mathfrak{R}$-class. Under the standing hypothesis, $h b=\theta N$, and as $b<N$, we have that $h>\theta$. Hence $\mathrm{c}\left(c_{N} N\right)=h$.

If $\delta \neq 0$, then $c_{b}=h$. Hence $c_{b} b=c_{N} N$. The factorizations of $c_{a} a$ are $(l, 0,0)$ and $(0, y+i h, \delta-i \theta), 0 \leqslant i \leqslant \frac{\delta}{\theta}$. As $h>\theta$, the element with least length in the $\mathfrak{R}$-class of $(0, y, \delta)$ is $(0, y, \delta)$ itself. In view of Lemma $5, \mathrm{c}\left(c_{a} a\right)=l$. One of the $\mathfrak{R}$-classes of $c_{b} b$ is $\{(0, h, 0)\}$ and the other is the one containing $(0,0, \theta)$. This $\mathfrak{R}$-class might also contain elements of the form $(i l, 0, \theta-i \delta)$ if $y=0$ and $\delta<\theta$; however these have length $\theta+i(l-\delta) \geqslant \theta$. As $h>\theta, \mathrm{c}\left(c_{b} b\right)=h$.

If $y=0$ and $\delta=\theta$, then $c_{a} a=c_{b} b=c_{N} N$. The factorizations of $c_{a} a$ are $(l, 0,0)$, $(0, h, 0),(0,0, \delta)$. As $\delta<h<l(N>b>a), \mathrm{c}(S)=l=\max \{h, l\}$.

2) In this case $\delta \neq 0, c_{b}=h$ and $c_{N}=\delta$.

If $\theta=0$, then $c_{a}=w$ and $c_{a} a=c_{b} b$. The set of factorizations of $c_{a} a$ is $\{(w, 0,0)$, $(0, h, 0)\}$. As $w a=h b$ and $a<b, w>h$ and $\mathrm{c}\left(c_{a} a\right)=w$. The $\mathfrak{R}$-classes of $c_{N} N$ are $\{(0,0, \delta)\}$ and $\left\{(l-i w, i h, 0) \mid 0 \leqslant i \leqslant \frac{l}{w}\right\}$. Any factorization in the second $\mathfrak{R}$-class has largest length that $\delta$, since $N>b>a$, and the least length of these factorizations is $l+\left\lfloor\frac{l}{w}\right\rfloor(h-w)$, because in this setting, as pointed out above, $h<w$.

If $\theta$ is nonzero, $c_{a}=l$ and $c_{a} a=c_{N} N$. We may assume that $w \neq 0$, since this case has been already studied. Clearly, $\mathcal{F}\left(c_{a} a ; S\right)=\{(l, 0,0),(0,0, \delta)\}$ and $\mathrm{c}\left(c_{a} a\right)=l$. The factorizations of $c_{b} b$ are $(0, h, 0)$ and $(w+i l, 0, \theta-i \delta), 0 \leqslant i \leqslant \frac{\theta}{\delta}$. Observe that $|(w+i l, 0, \theta-i \delta)|=w+\theta+i(l-\delta)>|(w, 0, \theta)|$, and $(w, 0, \theta)$ is $\mathfrak{R}$-related to $(w+i l, 0, \theta-i \delta)$. Hence $\mathrm{c}\left(c_{b} b\right)=\max \{w+\theta, h\}$.

3) If $\delta=0$ (by Lemma 2, $\theta \neq 0$ ), then $c_{a}=l, c_{b}=y$ and $c_{N}=\theta$. Thus $c_{a} b=c_{b} b$ and $\mathcal{F}\left(c_{a} a ; S\right)=\{(l, 0,0),(0, y, 0)\}$. This implies $\mathrm{c}\left(c_{a} a\right)=l$. The factorizations of $c_{N} N$ are $(0,0, \theta),(l-w, h-y, 0)$ and possibly some of the form $(i l-w, h-i y, 0)$. These are $\mathfrak{R}$-related to $(l-w, h-y, 0)$ and have larger lengths. Thus $\mathrm{c}\left(c_{N} N\right)=$ $\max \{\theta, l-w+h-y\}=l-w+h-y$ (by Lemma 5).

If $\theta=0$, arguing as in the case $y=0=\theta$, we obtain that $\mathrm{c}(S)=\max \{w, l-y+$ $\left.\left\lfloor\frac{l}{w}\right\rfloor(h-w)\right\}$.

Finally, if $\delta \theta \neq 0, \mathcal{F}\left(c_{N} N ; S\right)=\{(0,0, \delta+\theta),(l-w, h-y, 0)\}$. Thus, c $((\delta+$ $\theta) N)=l-w+h-y$. Analogously $\mathcal{F}\left(c_{b} b ; S\right)=\{(0, h, 0),(w, 0, \theta)\}$ and $\mathcal{F}\left(c_{a} a ; S\right)=$ $\{(l, 0,0),(0, y, \delta)\}$. From this we obtain that $\mathrm{c}(h b)=\max \{h, \theta+w\}$ and $\mathrm{c}(l a)=l$.

Example 6 Let $S=\langle 10,15,18\rangle$. Let us compare the procedure given in the last proposition with that of $[6]$. Since $(l, h, w, y, \delta, \theta)=(9,2,3,0,5)$, we get that $\mathrm{c}(S)=$ 
$\max \{3,9+\lfloor 9 / 3\rfloor(2-3)\}=6$. According to [6], in order to calculate this amount, we must compute those elements having more than one $\mathfrak{R}$-class. In our setting $c_{a}=3$, $c_{b}=2$ and $c_{N}=5$. Thus the only elements with more than one $\mathfrak{R}$-class are $30=$ $3 \times 10=2 \times 15$ and $90=5 \times 18$ (this can be also done by using Apéry sets as explained in [6, Corollary 3]). The set of factorizations of 30 and 90 are $\{(0,2,0),(3,0,0)\}$ and $\{(0,0,5),(0,6,0),(3,4,0),(6,2,0),(9,0,0)\}$, respectively. The two $\mathfrak{R}$-classes of 30 are $\{(0,2,0)\}$ and $\{(3,0,0)\}$. The $\mathfrak{R}$-classes of 90 are $\{(0,0,5)\}$ and $\{(0,6,0),(3,4,0),(6,2,0)$, $(9,0,0)\}$. Thus by taking in each $\Re$-class the factorization with least length, and then the maximum of these lengths, we obtain that $\mathrm{c}(S)=6$ (achieved in the $\mathfrak{R}$-class $\{(0,6,0)$, $(3,4,0),(6,2,0),(9,0,0)\}$ of 90$)$.

Example 7 Let $S=\langle 4,9,11\rangle$, then $(l, h, w, y, \delta, \theta)=(5,3,4,1,1,1)$. Hence

$$
\mathrm{c}(S)=\max \{l, w+\theta, h, l-w+h-y\}=\max \{5,5,3,3\}=5 .
$$

This computation can be compared with [6, Example 4].

\section{Applications}

In this work, two kinds of applications can be considered: numerical and symbolical ones. Given $S=\langle a, b, N\rangle$ and a related tile $\mathcal{H}$, the basic coordinates of any equivalence class $[m]_{N}$ inside $\mathcal{H}$ can be computed in $O(\log N)$-time complexity in the worst case (see [1] for more details). Therefore, there are some computations that can be done efficiently. For instance, the question " $m \in S$ ?", the computation of the basic factorization and the catenary degree $\mathrm{c}(S)$ can be done in $O(\log N)$, in the worst case. Hence, the expressions for $\mathrm{d}(m ;\langle a, b\rangle)$ and $\mathcal{F}(m ;\langle a, b\rangle)$ of Corollary 5 can be computed in $O(\log N)$ as well.

Example 8 Let us consider $S_{t}=\left\langle 3^{t}, 5^{t}, 7^{t}\right\rangle$ for $t \geqslant 1$ and $m_{t}=\mathfrak{f}\left(S_{t}\right)+1 \in S_{t}$. We compare the execution time of computing one factorization of $m_{t}$ in $S_{t}$. We use the command FrobeniusSolve $\left[\left\{3^{t}, 5^{t}, 7^{t}\right\}, m_{t}, 1\right]$ included in the Mathematica 6 package and our algorithm for the basic factorization (the basic coordinates have been computed following the algorithm [1]) implemented in Mathematica 6 as well. The time, in seconds, has been computed using the command AbsoluteTiming. Some figures are listed in Table 1 with entries: $t, d$ the number of digits of $m_{t}$, time of Mathematica 6's algorithm A, time of our algorithm B and the denumerant $\mathrm{d}\left(m_{t} ; S_{t}\right)$. The computation have been made in a netbook at $1.6 \mathrm{Gh}$.

The time of Algorithm A for $t=10$ is less than the time for $t=5$. It is strange behaviour, perhaps the command FrobeniusSolve uses several algorithms. When $t=80$, the calculation using Algorithm A has been aborted after 400 minutes of running time.

Expressions for $\mathrm{d}(m ;\langle a, b, N\rangle)$ and $\mathcal{F}(m ;\langle a, b, N\rangle)$ in Theorem 2 have linear-time complexity in $\mathrm{d}(m ;\langle a, b, N\rangle)$, that can be unavailable for many instances of $m$ and $N$. However these expressions can be very useful from the symbolical point of view, that is: given a sequence of 3-numerical semigroups $S_{t}=\left\langle a_{t}, b_{t}, N_{t}\right\rangle$, for $t \geqslant t_{0}$, and a sequence of natural 


\begin{tabular}{|r|r|r|r|c|}
\hline$t$ & $d$ & Algorithm A & Algorithm B & $\mathrm{d}\left(m_{t} ; S_{t}\right)$ \\
\hline \hline 5 & 6 & 0.091484 & 0.002205 & 2 \\
\hline 10 & 11 & 0.012437 & 0.005495 & 2 \\
\hline 20 & 21 & 0.668541 & 0.008313 & 1 \\
\hline 40 & 41 & 47.009083 & 0.010841 & 1 \\
\hline 80 & 82 & $>400 \times 60$ & 0.022728 & 1 \\
\hline
\end{tabular}

Table 1: Execution time for several values of $t$

values $\left\{m_{t}\right\}_{t \geqslant t_{0}}$, find closed formulae for $\mathrm{d}\left(m_{t} ; S_{t}\right), \mathcal{F}\left(m_{t} ; S_{t}\right)$ and the catenary degree $\mathrm{c}\left(S_{t}\right)$. These symbolic problems can be particular sequences, or some property of them, that have some interest.

As an example of what we are refering to, let us consider the upper bound for the Frobenius Number $G(N)=\max _{2 \leqslant a<b<N} \mathfrak{f}(a, b, N)$. Lewin found $G(N)$ in his work [15] in 1972 ,

$$
G(N)=\max _{\substack{0<a<b<N \\ \operatorname{gcd}(a, b, N)=1}} \mathfrak{f}(a, b, N)= \begin{cases}(N-2)^{2} / 2-1, & \text { if } N \text { is even } \\ (N-1)(N-3) / 2-1, & \text { if } N \text { is odd }\end{cases}
$$

The critical semigroups are $\langle N-2, N-1, N\rangle$ and $\langle N / 2, N-1, N\rangle$ when $N$ is even and $\langle(N-1) / 2, N-1, N\rangle$ when $N$ is odd. Let us consider $S_{N}=\langle N-2, N-1, N\rangle$ for even $N \geqslant 6$, the only 3-generated critical one. The two largest gaps of $S_{N}$ are $F_{N}=(n-$ $2)(N-2)-1$ and $G(N)=(n-1)(N-2)-1$, where $n=N / 2$.

Proposition 6 Set $S_{N}=\langle N-2, N-1, N\rangle$ for even $N=2 n \geqslant 6, F_{N}=(n-2)(N-$ $2)-1, G(N)=(n-1)(N-2)-1$ and $\mathrm{d}\left(m^{*} ; S_{N}\right)=\max _{F_{N}<m<G(N)} \mathrm{d}\left(m ; S_{N}\right)$. Then

$$
\mathrm{d}\left(m^{*} ; S_{N}\right)= \begin{cases}N / 4, & \text { when } N / 2 \text { is even } \\ (N-2) / 4, & \text { when } N / 2 \text { is odd }\end{cases}
$$

and

- for even $n, m^{*}=(n-2)(N-1)$ and

$$
\mathcal{F}\left((n-2)(N-1) ; S_{N}\right)=\left\{\left(\frac{N-4}{4}-t, 2 t, \frac{N-4}{4}-t\right) \mid t=0, \ldots, \frac{N-4}{4}\right\},
$$

- for odd $n, m^{*} \in\{(n-2)(N-1)-1,(n-2)(N-1),(n-2)(N-1)+1\}$ and

$$
\begin{aligned}
\mathcal{F}\left((n-2)(N-1)-1 ; S_{N}\right) & =\left\{\left(\frac{N-2}{4}-t, 2 t, \frac{N-6}{4}-t\right) \mid t=0, \ldots, \frac{N-6}{4}\right\}, \\
\mathcal{F}\left((n-2)(N-1) ; S_{N}\right) & =\left\{\left(\frac{N-6}{4}-t, 2 t+1, \frac{N-6}{4}-t\right) \mid 0 \leqslant t \leqslant \frac{N-6}{4}\right\}, \\
\mathcal{F}\left((n-2)(N-1)+1 ; S_{N}\right) & =\left\{\left(\frac{N-6}{4}-t, 2 t, \frac{N-2}{4}-t\right) \mid t=0, \ldots, \frac{N-6}{4}\right\} .
\end{aligned}
$$




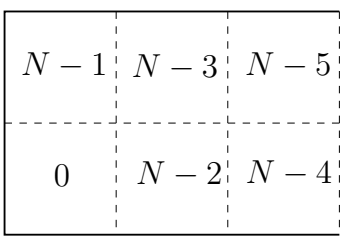

\begin{tabular}{|c|c|c|c|}
\hline 5 & 3 & 1 \\
& & & \\
\hline & 6 & 4 & 2 \\
& & & \\
\hline
\end{tabular}

Figure 6: $\mathcal{H}_{N}=L(n, 2,1,0)$ and the distribution of equivalence classes inside $\mathcal{H}$

Proof: By using the characterization given in [3], it can be checked that the tile $\mathcal{H}_{N}=$ $L(n, 2,1,0)$ is related to $S_{N}$. Then, we have $\delta_{N}=n-1$ and $\theta_{N}=1$.

Let us parameterize the values of $m, F_{N}<m<G(N)$, with the parameter $k$. Set $m_{k}=F_{N}+k=(n-2)(N-2)+k-1$ with $k=1, \ldots, G(N)-F_{N}-1=N-3$. Consider odd and even values of $k$ following the distribution of the equivalence classes modulo $N$ inside the tile $\mathcal{H}_{N}$. Note this distribution in Figure 6 . Then $m_{2 \ell+1} \equiv 2 \ell+4(\bmod N)$ for $\ell=0, \ldots, n-2$ and $m_{2 u} \equiv 2 u+3(\bmod N)$ for $u=1, \ldots, n-2$.

Let us study the odd and even cases of $k$ :

- $k=2 \ell+1$. The basic coordinates of $m_{2 \ell+1}$ are $\left(x_{0}, y_{0}\right)=(n-2-\ell, 0)$. Then, the value of $z_{0}$ is

$$
z_{0}=\frac{1}{N}[(n-2)(N-2)+2 \ell-(n-2-\ell)(N-2)]=\ell,
$$

thus $\left\lfloor\frac{z_{0}}{\delta_{N}+\theta_{N}}\right\rfloor=\left\lfloor\frac{\ell}{n}\right\rfloor=0$ for $0 \leqslant \ell \leqslant n-2, S_{0}=\left\lfloor\frac{z_{0}}{\delta_{N}}\right\rfloor=\left\lfloor\frac{\ell}{n-1}\right\rfloor=0$ and

$$
T_{0}=\min \left\{\left\lfloor\frac{n-2-\ell}{1}\right\rfloor,\left\lfloor\frac{\ell}{1}\right\rfloor\right\}=\min \{n-2-\ell, \ell\}= \begin{cases}\ell, & \ell \leqslant \frac{n-2}{2}, \\ n-2-\ell, & \ell>\frac{n-2}{2} .\end{cases}
$$

Therefore,

$$
n=2 p \Rightarrow T_{0}= \begin{cases}\ell, & \ell \leqslant \frac{n-2}{2} \\ n-2-\ell, & \ell \geqslant \frac{n-2}{2}+1,\end{cases}
$$

and

$$
n=2 p+1 \Rightarrow T_{0}= \begin{cases}\ell, & \ell \leqslant \frac{n-3}{2} \\ n-2-\ell, & \ell \geqslant \frac{n-3}{2}+1 .\end{cases}
$$

Hence, using the expression given in Theorem 2,

$$
n=2 p \Rightarrow \mathrm{d}\left(m_{2 \ell+1} ; S_{N}\right)= \begin{cases}1+\ell, & \ell \leqslant \frac{n-2}{2}, \\ n-1-\ell, & \ell \geqslant \frac{n-2}{2}+1,\end{cases}
$$

and

$$
n=2 p+1 \Rightarrow \mathrm{d}\left(m_{2 \ell+1} ; S_{N}\right)= \begin{cases}1+\ell, & \ell \leqslant \frac{n-3}{2}, \\ n-1-\ell, & \ell \geqslant \frac{n-3}{2}+1 .\end{cases}
$$

Clearly, the maximum value of the denumerant is attained at $\ell=\frac{n-2}{2}$ when $n=2 p$, and at $\ell=\frac{n-3}{2}, \frac{n-3}{2}+1$ when $n=2 p+1$. That is, when $n=2 p$,

$$
\mathrm{d}\left((n-2)(N-1) ; S_{N}\right)=p=\frac{N}{4}=\max _{0 \leqslant \ell \leqslant n-2} \mathrm{~d}\left(m_{2 \ell+1} ; S_{N}\right)
$$


and, for $n=2 p+1$,

$$
\begin{aligned}
\mathrm{d}\left((n-2)(N-1)-1 ; S_{N}\right) & =\mathrm{d}\left((n-2)(N-1)+1 ; S_{N}\right)= \\
& =p=\frac{N-2}{4}=\max _{0 \leqslant \ell \leqslant n-2} \mathrm{~d}\left(m_{2 \ell+1} ; S_{N}\right) .
\end{aligned}
$$

- $k=2 u$. The maximum denumerant is attained only when $n=2 p+1$ at $u=p$,

$$
\mathrm{d}\left((n-2)(N-1) ; S_{N}\right)=p=\frac{N-2}{4}=\max _{1 \leqslant u \leqslant n-2} \mathrm{~d}\left(m_{2 u} ; S_{N}\right)
$$

and, for $n=2 p$, we have $\max _{1 \leqslant u \leqslant n-2} \mathrm{~d}\left(m_{2 u} ; S_{N}\right)<N / 4$.

Therefore

$$
\max _{F_{N}<m<G(N)} \mathrm{d}\left(m ; S_{N}\right)= \begin{cases}N / 4, & \text { when } N / 2 \text { is even, } \\ (N-2) / 4, & \text { when } N / 2 \text { is odd, }\end{cases}
$$

and the critical values of $m$ are $m^{*}=(n-2)(N-1)$ when $n$ is even and $m^{*}=(n-$ $2)(N-1)-1,(n-2)(N-1),(n-2)(N-1)+1$ for odd $n$.

Following the generic parameterization $\mathcal{F}(m ;\langle a, b, N\rangle)$ in Theorem 2, we obtain the factorization sets of $m^{*}$ : for even $n$, we obtain the set

$$
\mathcal{F}\left((n-2)(N-1) ; S_{N}\right)=\left\{\left(\frac{N-4}{4}-t, 2 t, \frac{N-4}{4}-t\right) \mid t=0, \ldots, \frac{N-4}{4}\right\}
$$

and for odd $n$ it follows that

$$
\begin{aligned}
\mathcal{F}\left((n-2)(N-1)-1 ; S_{N}\right) & =\left\{\left(\frac{N-2}{4}-t, 2 t, \frac{N-6}{4}-t\right) \mid t=0, \ldots, \frac{N-6}{4}\right\}, \\
\mathcal{F}\left((n-2)(N-1) ; S_{N}\right) & =\left\{\left(\frac{N-6}{4}-t, 2 t+1, \frac{N-6}{4}-t\right) \mid t=0, \ldots, \frac{N-6}{4}\right\}, \\
\mathcal{F}\left((n-2)(N-1)+1 ; S_{N}\right) & =\left\{\left(\frac{N-6}{4}-t, 2 t, \frac{N-2}{4}-t\right) \mid t=0, \ldots, \frac{N-6}{4}\right\} .
\end{aligned}
$$

Note that the mean value $(n-2)(N-1)=\frac{F_{N}+G(N)}{2}$ is a critical value always.

Corollary 7 Using the notation of Proposition 6, the set $\left\{\mathrm{d}\left(m ; S_{N}\right) \mid F_{N}<m<G(N)\right\}$, for even $N$, has an unimodal distribution.

Proof: The unimodal distribution of $\mathrm{d}\left(m_{2 \ell+1} ; S_{N}\right)$, for $\ell=0, \ldots, N / 2-2$, is derived from (5) and (6). The unimodal distribution of $\mathrm{d}\left(m_{2 u} ; S_{N}\right)$, for $u=1, \ldots, N / 2-2$, results from an analogous reasoning.

The particular cases $N=20$ and $N=22$ are shown in Figure 7. For a fixed $N=N_{0}$, the values $\mathrm{d}\left(m, S_{N_{0}}\right)=p_{N_{0}}(m)$ can also be derived from the Ehrhart's quasipolynomials [10], by using Barvinok's algorithm [4]. These quasipolynomials are related to counting lattice points in polyhedra, that turns out to be equivalent to computing the denumerants $p_{N_{0}}(m)$. For instance when $N_{0}=22\left(S_{22}=\langle 20,21,22\rangle\right)$, the value of the denumerant 


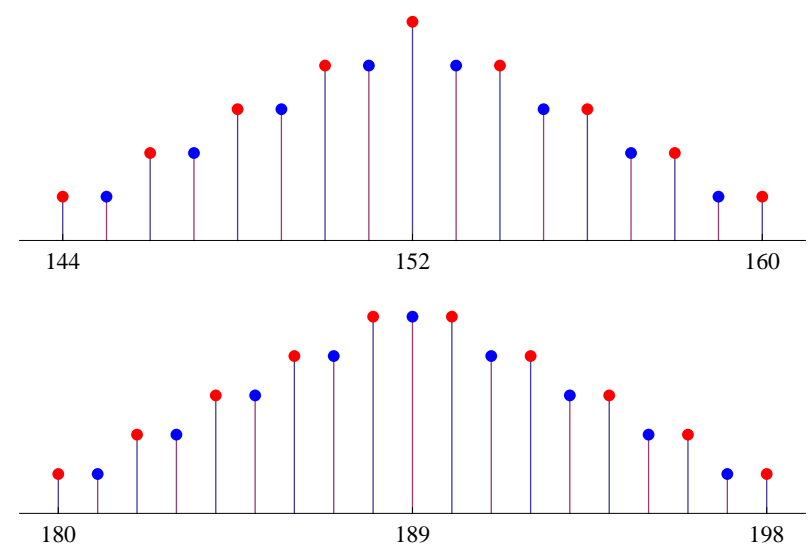

Figure 7: Unimodal distributions for $N=20$ and $N=22$

$\mathrm{d}\left(m, S_{22}\right)$ is the same as the number of lattice points $(x, y, z) \in \mathbb{N}^{3}$ in the triangle contained in the plane $20 x+21 y+22 z=m$, defined by the constraints $x \geqslant 0, y \geqslant 0$ and $z \geqslant 0$. This number, $p_{22}(m)$, is the related quasipolynomial given by the expression

$$
\begin{aligned}
p_{22}(m)= & \frac{1}{18480} m^{2}+\frac{1}{220}\left(1-\left\{\frac{m}{2}\right\}\right) m-\frac{355}{1848}-\frac{11}{2}\left\{\frac{m+21}{22}\right\}^{2} \\
& +\left(6-\left\{\frac{m}{2}\right\}\right)\left\{\frac{m+21}{22}\right\}+\frac{21}{2}\left\{\frac{m+20}{21}\right\}^{2}-\frac{19}{2}\left\{\frac{m+20}{21}\right\} \\
& -5\left\{\frac{m}{20}\right\}^{2}+\left(\left\{\frac{m}{2}\right\}+4\right)\left\{\frac{m}{20}\right\}-\frac{1}{22}\left\{\frac{m}{2}\right\}
\end{aligned}
$$

where $\{t\}=t-\lfloor t\rfloor$.

Proposition 7 For even $N=2 n \geqslant 6$, we have c $(\langle N-2, N-1, N\rangle)=N / 2$.

Proof: By using the related tile $\mathcal{H}_{N}=L(n, 2,1,0)$ and Theorem 5, we have

$$
\mathrm{c}\left(S_{N}\right)=\max \{n, 2,2\}=N / 2 .
$$

This value can also be computed following the work of Chapman, García-Sánchez and Llena in [6].

Acknowledgements: The authors thank Günter Rote for his helpful comments. They also thank Víctor Blanco for his computation of the quasipolynomial appearing in (7). The authors also thank the unknown referee for her/his helpful comments.

\section{References}

[1] F. Aguiló and J. Barguilla, Computing coordinates inside an L-shaped tile, Actas de las IV JMDA, Ed. UdL, ISBN 978-84-8409-263-6 (2008) pp. 35-41. 
[2] F. Aguiló and P. A. García-Sánchez, Factorization and catenary degree in 3-generated numerical semigroups, ENDM Vol. 34 (2009) 157-161.

[3] F. Aguiló, A. Miralles and M. Zaragozá, Using Double-Loop digraphs for solving Frobenius' Problems, ENDM Vol. 24 (2006) 17-24.

[4] Barvinok, A.I., Polynomial time algorithm for counting integral points in polyhedra when the dimension is fixed. Math of Operations Research 19 (1994) 769-779.

[5] J.-C. Bermond, F. Comellas and D.F. Hsu, Distributed loop computer networks: A survey, J. Parallel Distrib. Comput. 24 (1995) 2-10.

[6] Chapman, S. T., P. A. García-Sánchez, D. Llena, The catenary and tame degree of a numerical semigroup, Forum Math. 21 (2009) 117-129.

[7] S. T. Chapman, P. A. García-Sánchez, D. Llena, V. Ponomarenko, and J. C. Rosales, The catenary and tame degree in finitely generated cancellative monoids, Manuscripta Math. 120 (2006) 253-264.

[8] Cheng Y. and Hwang F., Diameters of Weighted Double-Loop Networks, J. Algorithms 9 (1988) 401-410.

[9] M. Delgado, P. A. García-Sánchez and J. Morais, "numericalsgps": a GAP [12] package on numerical semigroups. (http://www.gap-system.org/Packages/numericalsgps.html).

[10] Ehrhart, E., Polynômes arithmétiques et méthode des polyèdres en combinatoire, International Series of Numerical Mathematics, Vol. 35, Birkhäuser Verlag, Basel, 1977.

[11] M.A. Fiol, J.L.A. Yebra, I. Alegre and M. Valero, A discrete optimization problem in local networks and data alignment, IEEE Trans. Comput. C-36 (1987) 702-713.

[12] The GAP Group. GAP - Groups, Algorithms, and Programming, Version 4.4, 2004. (http://www.gap-system.org).

[13] A. Geroldinger and F. Halter-Koch, Non-unique Factorizations: Algebraic, Combinatorial and Analytic Theory, Pure and Applied Mathematics, vol. 278, Chapman \& Hall/CRC, 2006.

[14] J. Herzog, Generators and relations of abelian semigroups and semigroup rings, Manuscripta Math. 3 (1970) 175-193.

[15] Lewin M., A bound for a solution of a linear diophantine problem, J. London Math. Soc. 6 (1972) 61-69.

[16] T. Popoviciu, Asupra unei probleme de patitie a numerelor, Acad. Republicii Populare Romane, Filiala Cluj, Studii si cercetari stintifice (Romanian) 4 (1953) 7-58.

[17] J.L. Ramírez Alfonsín, The Diophantine Frobenius Problem. Oxford Univ. Press (2005) Oxford. ISBN 0-19-856820-7 978-0-19-856820-9.

[18] Ø.J. Rødseth, On a linear diophantine problem of Frobenius, J. Reine Angewandte Math. 301 (1978) 171-178. 
[19] Rosales, J. C. and García-Sánchez, P. A., Numerical semigroups. Developments in Mathematics, 20. Springer (2009) New York, ISBN: 978-1-4419-0159-0.

[20] Wolfram Research Inc., Mathematica Version 6.0, Wolfram Research Inc., (2007) Champaign, Illinois.

[21] C.K. Wong and D. Coppersmith, A combinatorial problem related to multimode memory organizations, J. ACM 21 (1974) 392-402. 